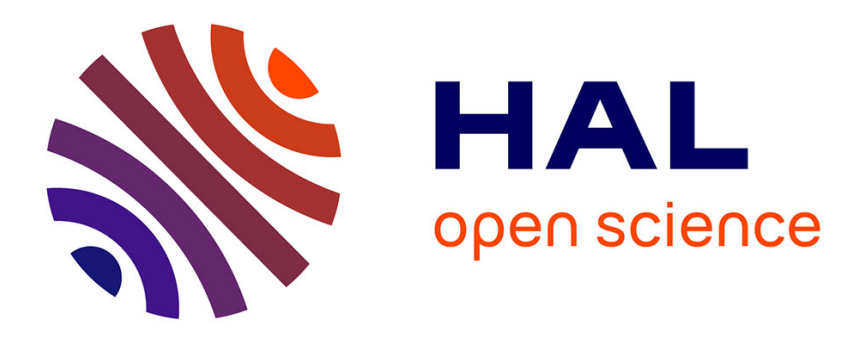

\title{
Overflow of Fountain Codes in Multi-hop Wireless Sensor Networks
}

Anya Apavatjrut, Katia Jaffres-Runser, Claire Goursaud, Cédric Lauradoux

\section{To cite this version:}

Anya Apavatjrut, Katia Jaffres-Runser, Claire Goursaud, Cédric Lauradoux. Overflow of Fountain Codes in Multi-hop Wireless Sensor Networks. IEEE 22nd Personal, Indoor and Mobile Radio Conference (PIMRC), IEEE, Sep 2011, Toromto, Canada. hal-00644445

HAL Id: hal-00644445

https://hal.inria.fr/hal-00644445

Submitted on 25 Nov 2011

HAL is a multi-disciplinary open access archive for the deposit and dissemination of scientific research documents, whether they are published or not. The documents may come from teaching and research institutions in France or abroad, or from public or private research centers.
L'archive ouverte pluridisciplinaire HAL, est destinée au dépôt et à la diffusion de documents scientifiques de niveau recherche, publiés ou non, émanant des établissements d'enseignement et de recherche français ou étrangers, des laboratoires publics ou privés. 


\title{
Overflow of Fountain Codes in Multi-hop Wireless Sensor Networks
}

\author{
Anya Apavatjrut, Katia E. Jaffrès-Runser, Claire Goursaud and Cédric Lauradoux \\ Université de Lyon, INRIA, \\ INSA-Lyon, CITI, F-69621, Villeurbanne, France \\ Email: surname.name@inria.fr
}

\begin{abstract}
This paper concentrates on the proper use of fountain codes for the transmission of sporadic data in a wireless sensor network (WSN). Fountain codes offer great perspectives for the self-organization of WSNs: they self adapt to the channel error rate without control packets. Deploying fountain codes in a WSN raises two problems. First, the size of the data transmitted by a sensor is small in comparison to the size usually considered with fountain codes. Second, WSNs mostly rely on multi-hop transmissions. It implies a non null transmission duration for the end-to-end acknowledgement of the reception. During this period of time, the source is still transmitting useless packets, creating a specific overhead we define as the overflow. This paper brings the overflow problem to light and analyses its impact on the network performance. Our work can be viewed as the networking counterpart of the results presented by Pakzad et al. at ISIT 2005 applied to WSNs.
\end{abstract}

\section{INTRODUCTION}

This paper is dedicated to the deployment of fountain codes in a wireless sensor network (WSN). A WSN is composed of sensor nodes with restricted capabilities (memory, energy and computational power) and a set of sink nodes which gather the sensed data. It is assumed here that the sink nodes have unlimited resources compared to the sensors. A direct communication between a source $\mathcal{S}$ (any sensor) and a destination $\mathcal{D}$ (any sink) is not always possible: relaying nodes $\mathcal{R}_{i}$ are used to carry the data following a hop-by-hop model. The cascade of $n$ channels [13] forms the main path and the reverse path forms the feedback path (Fig. 1). Such a cascade is established by a routing algorithm whose study is out of the scope of this paper.

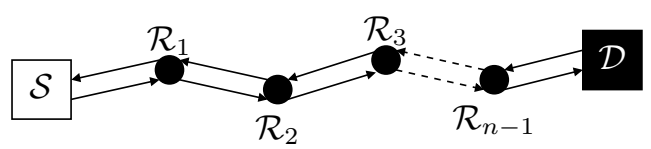

Fig. 1. A main and feedback path with $n$ hops.

In a WSN, the capacity of each channel in a path is unknown prior roll-out and even during the lifetime of the network: the nodes are usually scattered across the monitored area in a random way and the environment may vary. Fountain codes are a promising solution to provide robustness here [5], [14], [1], [6]. Indeed, they are rateless, i.e. a source $\mathcal{S}$ can potentially generate a limitless number of encoded packets until it receives an acknowledgement from $\mathcal{D}$. They can adapt to the channel condition on the fly. Another advantage of fountain codes over schemes such as automatic repeat request (ARQ) is the limited use of the feedback path. $\mathcal{D}$ only acknowledges a successful decoding to $\mathcal{S}$. This is why fountain codes have found important applications in satellite communications [4], content distribution [3] or underwater networking [5].

In order to take the full advantage of fountain codes in a WSN, it is mandatory to account for the characteristics of the data sent by $\mathcal{S}$ and of the feedback channel.

The data transmitted by a source $\mathcal{S}$ in a WSN depends on the target application. However, most of the time, sensors gather small amounts of data (e.g. temperature, light level or any physical quantity...) and send them at a regular or irregular pace to the sink $\mathcal{D}$. Hence, it is reasonable to consider that only few packets have to be sent at the same time, traffic is sporadic and data packets are short. This represents a major modification for the application of fountain codes. In previous applications [4], [3], large data are often assumed. The main impact of this change is the modification of the decoding overhead $\epsilon$ of most fountain codes (Raptor, LT...). Random linear codes are less affected by this modification and this is why they are used in this paper despite their high decoding complexity.

The feedback path from $\mathcal{D}$ to $\mathcal{S}$ does not allow to transmit instantaneously the acknowledgement to $\mathcal{S}$ at the end of the communication. While the acknowledgement is en route to $\mathcal{S}$, the fountain still flows, i.e. $\mathcal{S}$ and the $n-1$ relaying nodes $\mathcal{R}_{i}$ can still transmit data. This additional communication overhead is wasting the nodes resources. We call it the overflow by analogy to the amount of water wasted by a hosepipe before somebody in the garden can turn-off the tap.

The analysis of the overflow traffic depends on the size $k$ of the data to be transmitted and on the number of hops $n$. Indeed, the number of packets required to reach a successful decoding at $\mathcal{D}$ is lower bounded by $n(k+\epsilon)$. A simple model based on the acknowledgement progression gives us an overflow in $O\left(n^{2}\right)$ for a few relaying strategies. Therefore, the choices of $n$ and $k$ are critical to prevent the overflow to become dominant. Our analysis is supported by realistic simulations where both small scale fading and collisions for an unslotted ZigBee IEEE 802.15.4 medium access layer [17] are considered using the WSNet network simulator [7].

The main contribution of this paper is to bring to light the impact of the overflow on the overall network performance. We 
provide a first simple analytical model to quantify this overflow and highlight how various relaying strategies at the nodes can modify its magnitude in the network. Simulations using an IEEE 802.15.4 protocol stack exhibit how greedy random relaying is beneficial compared to basic passive relaying.

The rest of the paper is organized as follows. In the next section, existing works on fountain codes are summarized. In Section III, the overflow problem due to the hop-by-hop acknowledgement transmission is analyzed and section IV presents the impact of different relaying strategies on this overhead. Finally, Section V concludes the paper.

\section{FOUNTAIN CODES}

The concept of fountain codes was first presented in 1998 by Byers et al. [4]. Since then, many works have studied these topics. The design of a fountain code for a line network is composed of four tasks: (a) the encoding algorithm design, (b) the decoding algorithm design including the decoding overhead $\epsilon$, (c) the relaying strategy choice and (d) the overflow analysis. The first three tasks (a to c) are entwined and have been addressed in many works [3], [12], [16], [2], [9]. The design of efficient relaying strategies for fountain codes have recently deserved the attention of the communication community due to the development of network coding techniques [15], [1], [6]. To the knowledge of the authors, this paper is the first one addressing the overflow issue. In what follows, we briefly summarize tasks (a) to (c).

\section{A. Fountain encoding}

Given a set of $k$ input symbols, the source generates an infinite stream of output symbols which results from a linear combination of a subset of input symbols. For each encoded symbol, a degree $d \in[1, k]$ is chosen according to a degree distribution. Then, $d$ randomly chosen symbols are linearly combined over $\mathbb{F}_{2}$ to create an encoded symbol. This coding is repeated until an acknowledgement is received from $D$.

The degree distribution is the central parameter in the design of fountain codes that is linked to the decoding algorithm. The most simple distribution is the binomial one of random linear codes. Another possibility is the Robust Soliton distribution associated to LT-code introduced by Luby [12]. A modification of the encoding scheme of LT-code has been proposed by Shokrollahi with Raptor code [16]. A precode is applied on the $k$ input symbols prior to the fountain encoding. To conclude on the degree distribution, the results [9], [18] are worth mentioning. They study the optimal degree distribution that must be associated to a Gaussian elimination decoding for small value of $k$.

\section{B. Fountain decoding}

A reliable decoding algorithm for Fountain codes should allow the recovering of $k$ input symbols from any subset of $k+\epsilon$ output symbols with a probability that is at most inversely polynomial in $k$ [16].

The straightforward decoding algorithm consists in solving a system of $k+\epsilon$ equations using Gaussian elimination. This approach has a low decoding overhead, i.e. $\epsilon$ is $O\left(\frac{\log (k)}{k}\right)$ but a high decoding complexity $O\left(k^{3}\right)$ Later, Luby applied to its LT-codes [12] Belief Propagation (BP) decoding. It offers a better decoding complexity at the cost of decoding overhead (see Table I). Raptor code is an optimized version of LT code that combines an outer code to a regular LT code. Its decoding overhead is smaller than for LT ( $\epsilon=1$ or 2$)$ with the same decoding complexity (cf. [16] to get further details). $f$

\begin{tabular}{|c|c|c|c|}
\hline & Random Linear & LT & Raptor \\
\hline Decoding Overhead & $\frac{\log (k)}{k}$ & $\frac{\log ^{2}(k)}{\sqrt{k}}$ & 1 \\
\hline Encoding Complexity & $k^{2}$ & $k \log (k)$ & 1 \\
\hline Decoding Complexity & $k^{3}$ & $k \log (k)$ & $k$ \\
\hline
\end{tabular}

TABLE I

CHARACTERISTICS OF THE MAIN CLASSES OF FOUNTAIN CODES..

At ISIT 2009, Lu et al. [11] have introduced black-box linear algebra and more specifically the use of the Wiedemann algorithm for the decoding of fountain codes. This result offers great perspectives for both efficient decoding and a low $\epsilon$ when $k$ is small. However, this research area is still open and many works have still to be done in order to find optimal solutions.

The size of our problem (small values for $k$ ) raises two fundamental questions: $i$ ) are fountain codes beneficial for small values of $k$ ? and if so, $i i)$ what is the appropriate code (i.e. decoding algorithm) for this task? The reader is referred to [1] for the first question. The second question is meaningless since all decoding algorithms have roughly the same computation cost for small $k$.

\section{Relaying strategies}

The problem of relaying a fountain code over a line network was first studied by Pakzad et al. [15]. The most simple relaying strategy consists in not performing any processing. Throughout the paper, this strategy is referred to as passive relaying. It is well-known [19], [13] that if some processing is allowed at relaying nodes, the min-cut capacity can be achieved. The following strategies are considered.

In a decode-and-forward relaying, each intermediate node fully decodes and then re-encodes the information before forwarding packets to its neighbor. In this case, each transmitter (source or relay) compensates for the losses on the transmission link to its immediate neighbor.

The forward-and-decode relaying is an hybrid strategy between passive and decode-and-forward. A node $\mathcal{R}_{i}$ relays passively the messages and tries to decode at the same time. When it successfully decodes the information, it sends an acknowledgement to $\mathcal{R}_{i-1}$. After that, $\mathcal{R}_{i}$ becomes a new source and encodes information for the following nodes.

The greedy random relaying, as defined in [15], is considered as a form of network coding [8] where relays $\mathcal{R}_{i}$ forward random linear combinations over $\mathbb{F}_{2}$ of the received data.

\section{ACK AND OVERFLOW}

An important obstacle for the roll-out of fountain codes in a WSN is the cost of acknowledgement. The aim of this 
section is to provide an analysis of the overflow in an idealized communication scheme.

The cost of acknowledgement is related to two types of packets that are transmitted after a successful decoding by $\mathcal{R}_{i}$. Acknowledgment (ACK) packets are an incompressible cost: they are necessary to end the overall data transmission and for $\mathcal{S}$ to start transmitting other data. Since the source and the relays $\mathcal{R}_{i}$ are constantly transmitting encoded packets until an ACK message is being received, there is another overhead referred to as the overflow traffic. This overhead is measured by the number of the packets sent by $\mathcal{S}$ and the relaying nodes $\mathcal{R}_{i}$ after a successful decoding of the input packets by $\mathcal{D}$. They are a side-effect of a hop-by-hop mode of transmission of the ACK. Similarly, we denote in the following by acknowledgement traffic the number of ACK packets sent in the network. In our protocol, the destination sends an ACK after each received data packet in overflow (i.e. once decoding has been performed successfully).

To give an intuition of what are the acknowledgement and overflow traffic, we first consider transmission channels where no errors occur. Moreover, the message scheduling is supposed to be ideal. When the nodes $\mathcal{R}_{i-1}$ and $\mathcal{R}_{i+1}$ send simultaneously data to $\mathcal{R}_{i}, \mathcal{R}_{i}$ receives at least one message. Again, these assumptions are not made to express realistic transmissions but they are very useful to give a rough model of what happens after a successful decoding. Transmission delays along each channel of the main and feedback paths are assumed constant and identical for all packets.

The progression of the acknowledgement is $h$ times faster than the progression of the data: when a data packet progresses of one hop, the acknowledgement progresses of $h$ hops. This assumption is reasonable and is motivated by the fact that ACK packets are usually smaller than regular data packets, and hence suffer from a lower packet error rate (PER). Moreover, medium access protocols are often designed to prioritize the transmission of ACK packets in the network (with smaller inter frame separations in IEEE802.15.4 for instance). An example of transmission is given in Fig. 2 for $n=4$ and $h=1$.

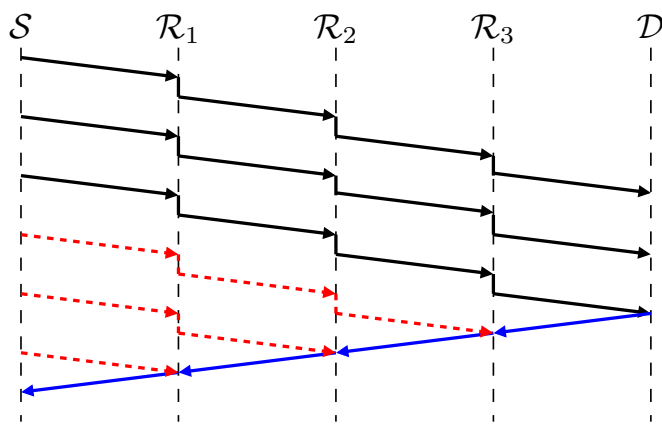

Fig. 2. Message timeline. The black plain arrows represent the transmission that carries useful information to $\mathcal{D}$. After and during a successful decoding all the transmissions are useless to $\mathcal{D}$ (dotted arrows). After a successful decoding, $\mathcal{D}$ acknowledges the information reception. The emission of the acknowledgement can collide with some emissions at the node level. It is assumed here for simplicity that collision are handled in favor to the ACK.
Over a line network, it is straightforward to see that for any relaying strategy, the acknowledgement traffic is at least linear in the number of hops $n$ since $\mathcal{S}$ and each relay $\mathcal{R}_{i}$ has to receive the ACK. For passive and greedy random relaying, the ACK is initialted by $\mathcal{D}$ and has to travel hop-by-hop to $\mathcal{S}$. For decode-and-forward and forward-and-decode relaying, the source $\mathcal{S}$ is virtually moving towards $\mathcal{D}: \mathcal{S}$ receives the ACK from $\mathcal{R}_{1}, \mathcal{R}_{1}$ from $\mathcal{R}_{2}$ and so on.

The overflow is in $O(n)$ for decode-and-forward. The node $R_{i}$ acknowledges $R_{i-1}$ and can only receive some overflow from $R_{i-1}$. The forward-and-decode is similar: if $\mathcal{D}$ decodes successfully, then $\forall i \in[1, n-1], \mathcal{R}_{i}$ has decoded successfully.

For passive and greedy random relaying, the overflow can be approximated by (in number of packets):

$$
n\left\lfloor\frac{n}{h}\right\rfloor-h \cdot \frac{\left\lfloor\frac{n}{h}\right\rfloor\left(\left\lfloor\frac{n}{h}\right\rfloor+1\right)}{2} .
$$

If the ACK has been received by $r$ nodes, there are still $(n-r h)$ potential nodes relaying information. Equation (1) is obtained by the summation of all the nodes that can potentially emit information before the ACK reaches $S$.

The model considered here is far from being realistic but it gives us a lower bound on the overflow. Next Section IV challenges these bounds for a 802.15.4 WSN.

\section{Simulation}

\section{A. Simulation setup}

\begin{tabular}{|l|l|}
\hline Layer & Configuration \\
\hline Application & $\begin{array}{l}\text { Network size (in hops): } n \\
\text { Distance between each node }=85 \mathrm{~m}\end{array}$ \\
\hline Networking & $\begin{array}{l}\text { MAC protocol: Unslotted IEEE 802.15.4 CSMA/CA } \\
\text { Transmission period of source }=1 \mathrm{~s} \\
\text { Coding: RL code } \\
\text { PDU size }=128 \text { bytes } \\
\text { Block length }=k\end{array}$ \\
\hline Radio & $\begin{array}{l}\text { Radio device: Chipset CC1100 } \\
\text { Modulation: BPSK } \\
\text { Transmitted power }=10 \mathrm{~dB} \\
\text { Transmission rate }=20 \mathrm{Kbit} / \mathrm{s} \\
\text { Frequency = 868MHz }\end{array}$ \\
\hline Propagation & $\begin{array}{l}\text { Propagation model: AWGN } \\
\text { Pathloss exponent } \alpha=2 \\
\text { White noise }=-111 \mathrm{dBm} / \mathrm{Hz} \\
\text { Fading: none }\end{array}$ \\
\hline
\end{tabular}

TABLE II

SIMULATION PARAMETERS.

Our simulations are done on WSNet [7], an event-driven network simulator. The MAC protocol used by the sensors follows the IEEE802.15.4 standard [17] and the physical characteristics correspond to the CC1100 Chipset from Texas Instruments [10]. The access method of IEEE 802.15.4 considered in this paper is unslotted CSMA/CA. An ACK packet is a regular data packet with a single bit payload. So far, most of the studies on fountain codes have assumed perfect feedback mechanisms. In this paper, we consider a realistic transmission scheme where acknowledgments can also suffer from loss based on various channel statistics. However, even 
in a realistic configuration, ACK packets have a lower PER because of their smaller size. In our configuration, $\mathcal{D}$ repeats the acknowledgment until it stops receiving data packets. The

\begin{tabular}{|l|c|c|c|}
\hline & $n=5$ & $n=10$ & $n=15$ \\
\hline Decode-and-forward & 69.9 & 138.2 & 206.5 \\
\hline Forward-and-decode & 66.8 & 134.1 & 202.7 \\
\hline Passive relaying & 24.4 & 53.0 & 111.3 \\
\hline Greedy random relaying & 20.2 & 29.0 & 57.9 \\
\hline \multicolumn{4}{|c|}{ TABLE III } \\
TRANSMISSION DELAY IN SECONDS FOR $k=10$.
\end{tabular}

transmission channel is characterized by its PER derived as a function of the Signal to Noise Ratio (SNR) $\gamma$ on a link. The SNR is derived using an isotropic propagation model with a pathloss exponent of $\alpha=2$. The PER depends on Bit Error Rate (BER) as follows: $P E R=1-(1-B E R(\gamma))^{\ell}$, with $\ell$ the length of the packet. The BER depends on the type of modulation and the type of channel considered. It is derived in our case for a BPSK modulation and AWGN channel model using $B E R(\gamma)=Q(\sqrt{2 \gamma})=0.5 * \operatorname{erfc}(\sqrt{\gamma})$.

The parameters used in our simulations are summarized in Table II. The simulation results are averaged over 1000 trials. A first simulation set deals with the transmission delay of the different strategies for $k=10$ in Table III. The best solution is greedy random relaying: it is fast and scales well. At the other end, decode-and-forward is the slowest and its delay grows linearly with $n$.

We now turn our attention to characterizing overflow.

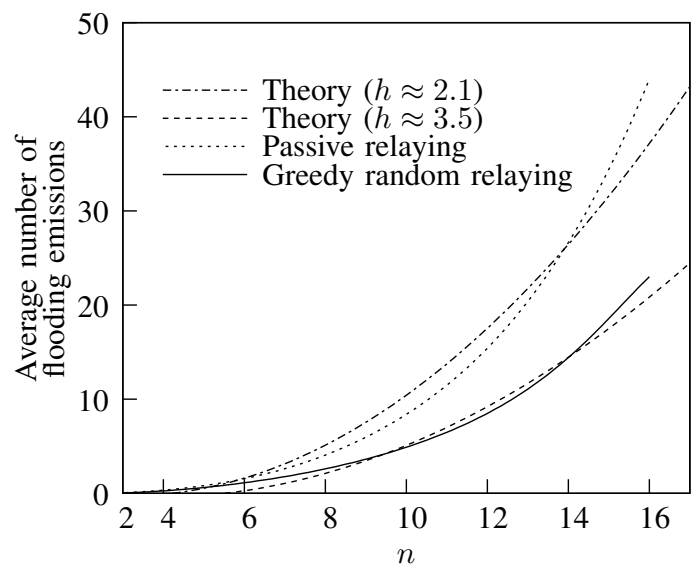

Fig. 3. Validation of the overflow for passive and greedy random in average.

\section{B. Assessing the overflow model}

The overflow has been computed as a function of $n \in$ $[2,15]$, for $k=10$. The overflow observed for decodeand-forward and forward-and-decode is marginal. For passive and greedy random relaying, the analytical estimation of Equation (1) is confronted with our simulations in Fig. 3 on average results. Parameter $h$ for each strategy has been obtained through a linear regression method. It appear that the model matches relatively well the simulation. Passive relaying is more impacted by the overflow than greedy random is.

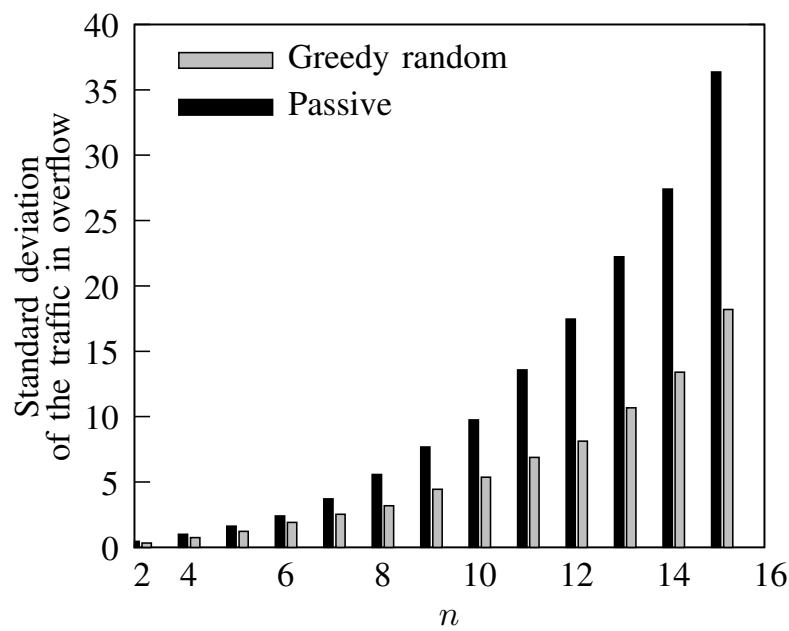

Fig. 4. Standard deviation of the overflow.

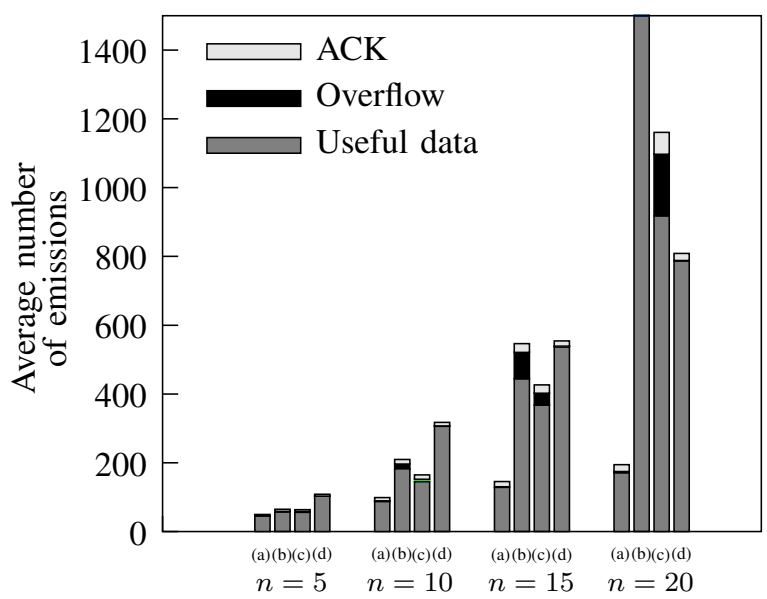

Fig. 5. Traffic decomposition of decode-and-forward (a), passive relaying (b), greedy random (c) and forward-and-decode (d) relaying for $n=$ $\{5,10,15,20\}$ and $k=5$.

We observe a high variability in our results. The standard deviation in our previously mentioned simulation results grows quickly with $n$ (see Fig. 4). We observe with nearly the same probability trials in which $h \approx n$ or $h \approx 1$. This is entirely due to random backoff of 802.15.4 that induces high variability in the medium access. We ensure that no congestion occurs by setting the transmission period of the source to one second. The conclusion is that the choice of the MAC layer may greatly influence the overflow.

\section{Comparing the strategies}

Figure 5 provides a detailed traffic analysis of the different strategies for $n=\{5,10,15,20\}$ and $k=5$. In this dimension, decode-and-forward relaying offers the best results and a high scalability. Passive and greedy random relaying have an 


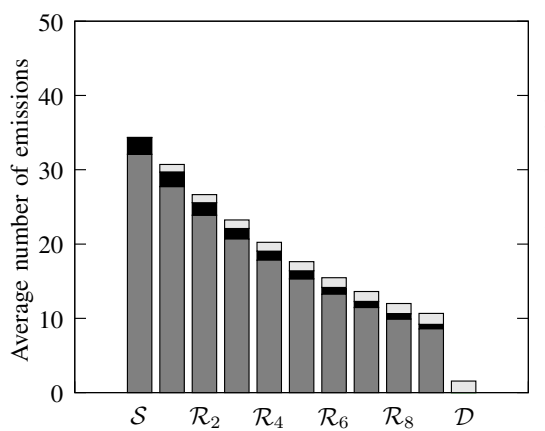

(a) passive

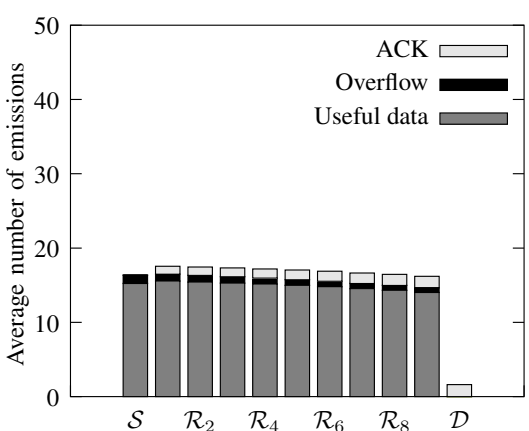

(b) Greedy random

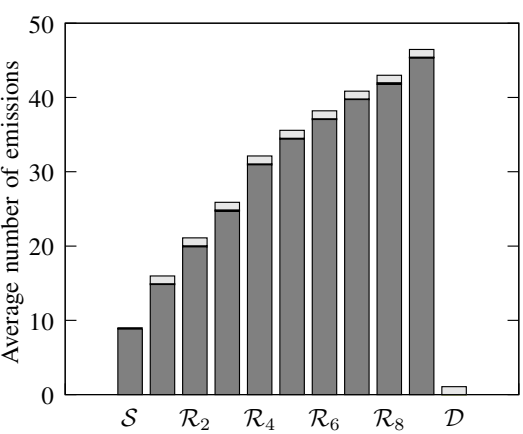

(c) forward-and-decode

Fig. 6. Emitted traffic for each node $(n=10)$. We have $k=5$ and we consider passive (left), greedy (center) and forward-and-decode (right) relaying.

exponential growth and for $n=20$, passive relaying is almost untractable. For $n=15$, the overflow represents $14 \%$ (resp. $8 \%$ ) of the overall traffic for passive relaying (resp. greedy random relaying).

The Fig. 6 (a) to (c) show the traffic emitted by each relay relative to passive, greedy random and forward-and-decode strategies for $k=5$ and $n=10$. In this case, greedy random performs the best. It has also the best fairness (Jain's fairness equals to 0.92). Passive relaying and forward-and-decode have respectively a fairness of 0.80 and 0.79 . For forward-anddecode, many packets are redundant which explains the shape of the curve. Removing this redundancy is possible, but at the price of a quadratic encoding cost.

\section{CONCLUSION}

Fountain codes are attractive for WSNs because of their rateless property. Our analysis identified the main obstacles to their deployments on IEEE 802.15.4, i.e. the data size $k$ and the acknowledgement path ( $n$-hop). Decode-and-forward relaying is to be preferred if the application is delay-tolerant since it guarantees a minimal number of transmissions. It preserves the sensors' energy. For applications in which both delay and energy are critical, the situation is more complicated. If the number of hops on the route is below fifteen, greedy random relaying is well adapted. Otherwise, forward-anddecode is better. An important question left by the paper is how does it scale with other MAC layers. It is easy to see that our model applies well on TDMA schemes. Many MAC layers have been proposed to preserve the sensors energy through efficient duty-cycling. The authors will look next into the suitability of such solutions for deploying fountain codes.

\section{REFERENCES}

[1] Anya Apavatjrut, Claire Goursaud, Katia Jaffrès-Runser., Cristina Comaniciu, and Jean-Marie Gorce. Toward Increasing Packet Diversity for Relaying LT Fountain Codes in Wireless Sensor Networks. Communications Letters, IEEE, 15(1):52-54, January 2011.

[2] Amos Beimel, Shlomi Dolev, and Noam Singer. RT oblivious erasure correcting. IEEE/ACM Transactions on Networking, 15(6):1321-1332, 2007.

[3] John W. Byers, Michael Luby, and Michael Mitzenmacher. Accessing Multiple Mirror Sites in Parallel: Using Tornado Codes to Speed Up Downloads. In INFOCOM '99, pages 275-283, New York, NY, USA, 1999. IEEE.
[4] John W. Byers, Michael Luby, Michael Mitzenmacher, and Ashutosh Rege. A Digital Fountain Approach to Reliable Distribution of Bulk Data. In Conference on Applications, Technologies, Architectures, and Protocols for Computer Communication - SIGCOMM 1998, pages 5667, Vancouver, BC, Canada, August 1998.

[5] Paolo Casari, Michele Rossi, and Michele Zorzi. Fountain codes and their application to broadcasting in underwater networks: performance modeling and relevant tradeoffs. In ACM international workshop on Underwater Networks - WuWNeT '08, pages 11-18, San Francisco, California, USA, 2008. ACM.

[6] Mary-Luc Champel, Kévin Huguenin, Anne-Marie Kermarrec, and Nicolas Le Scouarnec. LT Network Codes. In International Conference on Distributed Computing Systems - ICDCS 2010, pages 536-546, Genoa, Italy, 21-25 June 2010.

[7] Antoine Fraboulet, Guillaume Chelius, and Eric Fleury. Worldsens: development and prototyping tools for application specific wireless sensors networks. In ACM/IEEE International Conference on Information Processing in Sensor Networks -IPSN 2007, pages 176-185. ACM, 2007.

[8] Christina Fragouli, Jean-Yves Le Boudec, and Jörg Widmer. Network coding: an instant primer. Computer Communication Review, 36(1):6368, 2006.

[9] Esa Hyytiä, Tuomas Tirronen, and Jorma T. Virtamo. Optimal Degree Distribution for LT Codes with Small Message Length. In INFOCOM 2007, pages 2576-2580, Anchorage, AK, USA, May 2007. IEEE.

[10] Texas Instruments. CC1100 Single Chip Low Cost Low Power RFTransceiver, 2006. http://focus.ti.com/lit/ds/symlink/cc1100.pdf.

[11] Feng Lu, Chuan Heng Foh, Jianfei Cai, and Liang-Tien Chia. LT codes decoding: Design and analysis. In International Symposium on Information Theory - ISIT 2009, pages 2492-2496, Seoul, South Korea, 2009. IEEE.

[12] Michael Luby. LT Codes. In Foundations of Computer Science FOCS 2002, pages 271-, Vancouver, BC, Canada, November 2002. IEEE Computer Society.

[13] Urs Niesen, Christina Fragouli, and Daniela Tuninetti. On Capacity of Line Networks. IEEE Transactions on Information Theory, 53(11):4039-4058, 2007.

[14] A. Oka and L. Lampe. Data extraction from wireless sensor networks using distributed fountain codes. IEEE Transactions on Communications, 57(9):2607-2618, 2009.

[15] Payam Pakzad, Christina Fragouli, and Amin Shokrollahi. Coding schemes for line networks. In IEEE International Symposium on Information Theory - ISIT 2005, pages 1853-1857, Adelaide, Australia, 2005. IEEE.

[16] Amin Shokrollahi. Raptor codes. IEEE Transactions on Information Theory, 52(6):2551-2567, 2006.

[17] IEEE Computer Society. IEEE Std 802.15.4 - Wireless Medium Access Control (MAC) and Physical Layer (PHY) Specifications for Low-Rate Wireless Personal Area Networks (LR-WPANs). Standard, 2003.

[18] Tuomas Tirronen. Fountain Codes: Performance Analysis and Optimisation. $\mathrm{PhD}$ thesis, 2010.

[19] Daniela Tuninetti and Christina Fragouli. On the throughput improvement due to limited complexity processing at relay nodes. In IEEE International Symposium on Information Theory - ISIT 2005, pages 1081-1085, Adelaide, Australia, 2005. IEEE. 